\title{
Article
}

\section{Anxiety, impaired social and aggressive behaviour correlates with cognitive and molecular changes in older SAMP8 males}

\author{
Foteini Vasilipoulou ${ }^{\sharp 1}$, Júlia Companys-Alemany ${ }^{* 1}$, Anna-Maria Canudas ${ }^{1}$, Verónica Palomera- \\ Ávalos², Daniel Ortuño-Sahagún ${ }^{3}$, Mercè Pallàs ${ }^{1, *}$ and Christian Griñán-Ferré ${ }^{1}$ \\ 1 Department of Pharmacology and Therapeutic Chemistry, Institut de Neurociències-Universitat de \\ Barcelona, Avda. Joan XXIII, 27. 08028 Barcelona, Spain; ftn.vasilopoulou@gmail.com (F.V.); \\ juliacompanysalemany@gmail.com (J.C.-A.); canudas@ub.edu (A.-M.C.); \\ christian.grinan@ub.edu (C.G.-F.) \\ 2 Department of Cellular and Molecular Biology, University Center of Biological and Agricultural Sciences, \\ University of Guadalajara, km 15.5 Guadalajara-Nogales highway, C.P. 45110 Zapopan, Jalisco, México. \\ vpalomera@hotmail.com (V.P.-Á.) \\ 3 Laboratorio de Neuroinmunología Molecular, Instituto de Investigación de Ciencias Biomédicas (IICB) \\ CUCS, Universidad de Guadalajara, Jalisco 44340, México. daniel.ortuno.sahagun@gmail.com (D.O.-S.) \\ \# Equal author contribution \\ * Correspondence: pallas@ub.edu; Tel.: +34 934024531
}

\begin{abstract}
Alzheimer's disease (AD) is characterized by cognitive impairment and different noncognitive deficits called "Behavioural and psychological symptoms of dementia" (BPSD) related to neurotrophin alterations, which differ from those presented in normal aging. Mouse models, both transgenics and inbreed mice models of $\mathrm{AD}$, are a useful tool in understanding the underlying mechanisms of the disease. The SAMP8 (senescence-accelerated mouse prone 8) mice line was generated from AKR/J strain by Professor Toshio Takeda at the University of Kyoto. This strain exhibited a particular early-onset and accelerated aging phenotype. The present study characterizes and provides information regarding the non-cognitive, cognitive and neurotrophin alterations and their correlation, demonstrating the AD-like symptoms presented in older males SAMP8. The cognitive impairment presented was accompanied by a reduction in sociability and an increase in aggressive as well as anxiety behaviours. Furthermore, changes in two of the most important neurotrophins, such as brain-derived neurotrophic factor (BDNF) and neurotrophin-3 (NT3) were found. Thus, the present results reveal new insights in this useful inbred mouse model of neurodegeneration and $\mathrm{AD}$, demonstrating the potential relationship between neurotrophin modifications, cognitive impairment and neuropsychiatric disorders (ND).
\end{abstract}

Keywords: behaviour; BPSD; cognitive decline; aging; correlations; SAMP8

\section{Introduction}

Aging has been strongly associated with a progressive loss of brain physiological integrity, leading to impaired function and increased vulnerability to death [1]. This brain deterioration is a key aspect for developing age-related diseases [2] and is commonly accompanied by cognitive decline and behavioural alterations, which can be classified as "Behavioural and psychological symptoms of dementia" (BPSD) or as neuropsychiatric symptoms (NPS) [3].

Advanced age or living longer is the most substantial risk factor for the development of some type of dementia [4]. Thus, Alzheimer's disease (AD), the leading cause of dementia in the elderly, is a progressive age-dependent neurodegenerative disease mainly characterized by cognitive decline and accompanied by a wide variety of non-cognitive neuropsychiatric disturbances [5]. The BPSD represent one of the most distressing manifestations of dementia and are widely accepted as hallmarks of $\mathrm{AD}$ and age-related dementias [6]. In $\mathrm{AD}$ patients, the manifestation of BPSD is 
differently influenced by the progression of dementia and by cognitive dysfunction [7,8]. Among BPSD, depression and anxiety are the most frequent neuropsychiatric symptoms in AD while aggressive and impulsive behaviours are of special significance with a direct influence on social functioning [9].

NPS and BPSD encompass a variety of symptoms such as hyperactivity, psychosis, affective disorder and apathy which represent a heterogeneous group of non-cognitive symptoms that not always appear in the same pattern, but that tends to occur together in syndromes or clusters, which can be, clinically, as relevant as cognitive decline [10]. The clinical importance of BPSD lies in their intimate relationship with $\mathrm{AD}$, since they can participate in its progression, increasing our knowledge in these symptoms we could be closer to effectively understand $\mathrm{AD}$ and its progression.

Advances in knowledge on the BPSD in the AD field are fundamental, given that the manifestation of BPSD impacts directly in the quality of patients' and their caregivers' quality lives [11]. In this context, animal models with such deficits are a useful tool to understand the pathology onset and development in gerontological research and for its progress on the treatment of dementia in humans. Several AD rodents models have been generated to predict and correlate different factors of the pathology to determine the causal factor. However, even though a variety of transgenic models have been produced for the study of learning/memory deficits, gene engineering methodology cannot be applied to BPSD problem given their uniqueness in dementia patients [7].

Insight into the mechanism factor underlying this variety of symptoms may be obtained by evaluating two of the most prominent members of neurotrophin families; brain-derived neurotrophic factor (BDNF) and neurotrophin-3 (NT3). It has been noted that AD patients characterized by BPSD, such as depression presented lower levels of BDNF throughout the central nervous system (CNS) [12]. Also, a slight reduction of NT3 in the cortex of AD patients have been described [13]. Furthermore, these neurotrophins have an important role as modulators of plasticity that is essential to regulate experience-dependent behaviour, confirming the participation in the BPSD.

The senescence-accelerated mouse 8 (SAMP8) is an accelerated aging model that was established through phenotypic selection from AKR/J mice by Takeda in early 1979 [14]. Due to the presence of AD-like cognitive and non-cognitive symptoms together with biochemical alterations, the SAMP8 model has been suggested as a model of accelerated age-related cognitive decline $[15,16]$. This mouse shows histopathological signatures of AD, such as abnormal APP and amyloid- $\mathrm{B}$ metabolism and oxidative damage as well as exhibits cognitive and emotional disturbances from young ages [17]. It is widely used as a for the investigation of the mechanism of age-related learning and memory deficits and as a therapeutic intervention model for AD and other dementias [18,19]. Furthermore, SAMP8 mice have been characterized by different learning and emotional tasks associated with circadian rhythms at different ages [20]. However, an extensive evaluation of the emotional behaviours with aging in SAMP8 mice and their implication in cognitive decline is still unknown. While it has been described that the altered anxiety state of SAMP8 mice is related to learning impairments [21,22] and reduced social interaction [23], a comprehensive characterization of aggression in regard to cognitive and non-cognitive symptoms, has not been reported in SAMP8 strain.

Specifically, this study examined different behavioural paradigms to detect the most important aspects of the non-cognitive phenotype in SAMP8 mice. Besides, we determined the cognitive state and some molecular parameters to establish the correlation among them. Overall, it was demonstrated that SAMP8 mice exhibit a robust correlation among neurotrophins changes and alterations in anxiety and emotional capabilities as well as aggressive and social responses when compared to the senescence-accelerated resistant 1 (SAMR1) age-matched healthy control strain (Figure 6). 


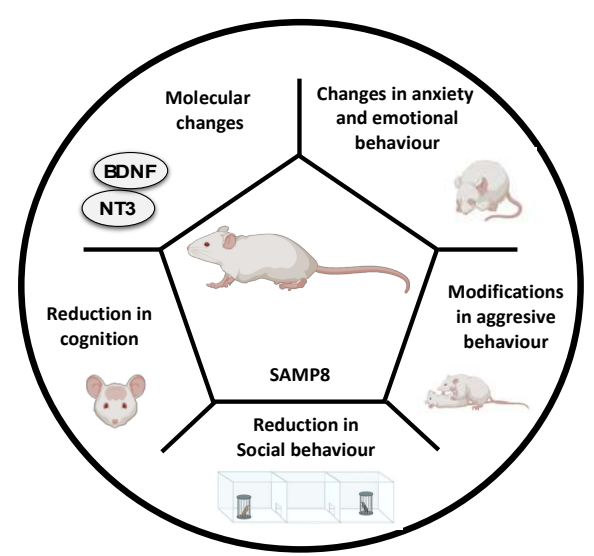

Figure 6. Illustrative cartoon of behavioural and cognitive changes as well as molecular alterations presented in SAMP8 elder male mice.

\section{Results}

\subsection{Increased anxiety-like behaviour in older SAMP8 male mice}

Emotional and anxiety-like behaviour parameters were evaluated by EPM and OFT. Our results revealed that SAMP8 group spent significantly more time in the open arms (Figure 1A) and less time in the center zone when compared to SAMR1 group (Figure 1B). Interestingly, the percentage of time spent in closed arms was also found slightly increased for the SAMP8 mice (Table S3). The vertical activity was also measured by counting the number of rearings done for each animal, and SAMP8 mice showed a significant decrease in this parameter compared to SAMR1 group (Figure 1C). Moreover, OFT was performed to further evaluate the anxiety-like behaviour and locomotor activity in the SAM strains. SAMP8 presented a significant increase in the total distance travelled in the open field arena compared to the SAMR1 group (Figure 1D). Accordingly, the vertical activity of the mice was evaluated, and it was found a significant increase in the number of rearings in SAMP8 group compared to the SAMR1 group (Figure 1E). Finally, SAMP8 mice spent significantly more time in the border area of the open field arena when compared to SAMR1 mice (Figure 1F) and tended to spend less time in the center zone (Table S4).
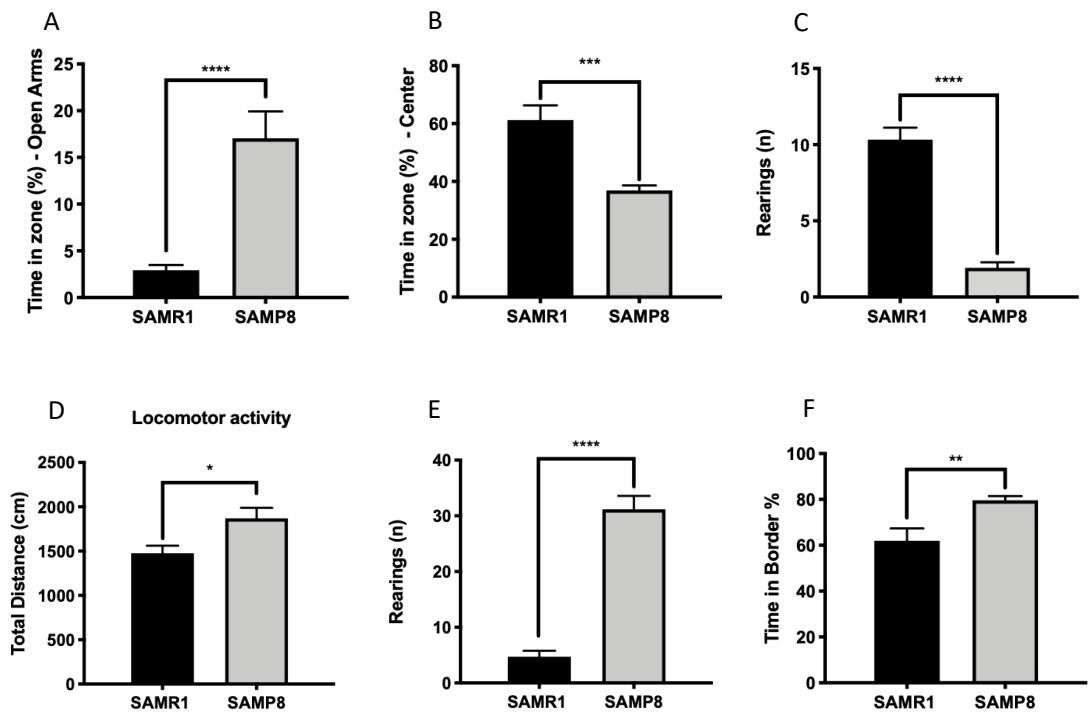

Figure 1. Results of the Elevated Plus Maze (EPM) and Open Field (OF) tests in males SAMR1 and SAMP8 mice at 12 months of age. For EPM: Time spent in open arms (A), time spent in center (B), 
number of rearings (C). For OF: Total distance (D), number of rearings (E) and time spent in Border zone $(\mathrm{F})$. Values represented are mean \pm Standard error of the mean $(\mathrm{SEM}) ; \mathrm{n}=26(\mathrm{SAMR} 1 \mathrm{n}=12$; SAMP8 $\mathrm{n}=14) .{ }^{*} \mathrm{p}<0.05 ;{ }^{* *} \mathrm{p}<0.01 ;{ }^{* * *} \mathrm{p}<0.001 ;{ }^{* * * *} \mathrm{p}<0.0001$.

\subsection{Impairment in social behaviour was present in aged SAMP8 male mice}

Sociable behaviour was assessed by TCT. No differences in the time spent in each chamber of the apparatus in the habituation phase was reported neither in the SAMP8 nor in the SAMR1 group, confirming the no-preference for any chamber (Figure 2A). When an intruder animal was incorporated in one chamber (sociability phase), SAMR1 group significantly spent more time into the intruder's chamber, whereas SAMP8 mice did not show any preference between the empty and the intruder's chamber (Figure 2B). In the same line, SAMP8 group spent significantly less time exploring the intruder mouse compared to SAMR1 mice (Figure 2C) revealing a relevant difference between strains in terms of sociability.
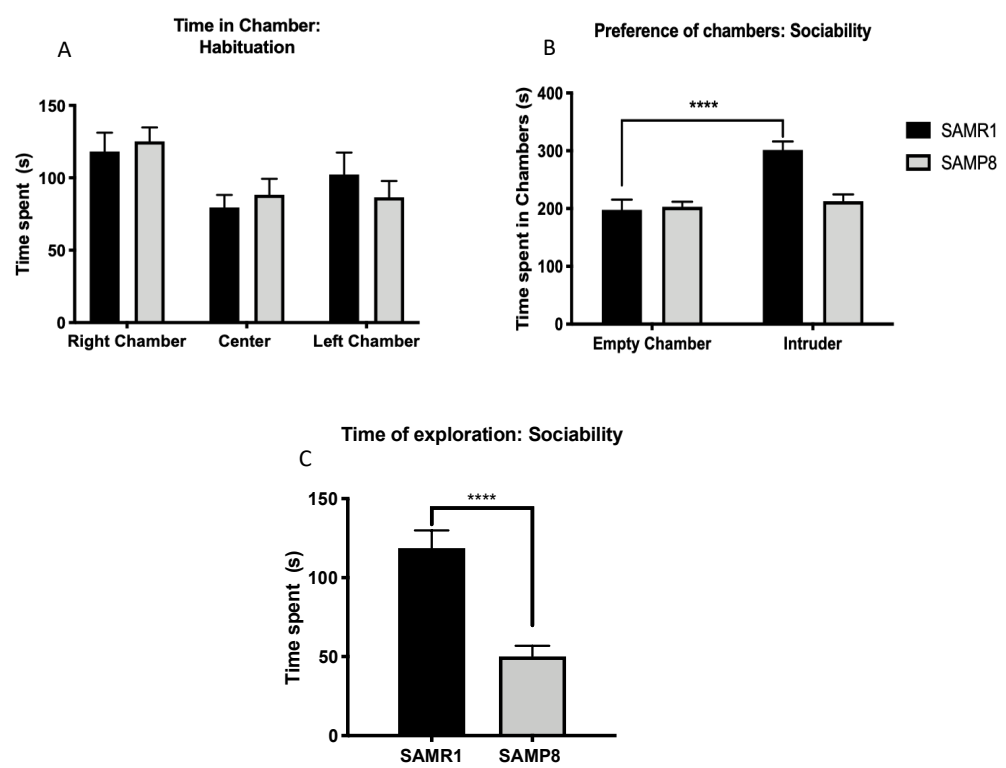

Figure 2. Results of Three Chamber Test (TCT) in males SAMR1 and SAMP8 mice at 12 months of age. Time spent in chambers during habituation (A), Time spent in chambers during sociability test (B) and time spent exploring intruder mouse $(C)$. Values represented are mean \pm Standard error of the mean (SEM); $\mathrm{n}=26$ (SAMR1 $\mathrm{n}=12$; SAMP8 $\mathrm{n}=14$ ). ${ }^{* * * *} \mathrm{p}<0.0001$.

\subsection{Increased aggressive behaviour exhibited by older SAMP8 male mice}

SAMP8 male mice presented increased aggressive behaviour when compared to SAMR1 mice as evaluated by RI. The latency to the first attacks against the intruder was found significantly decreased in SAMP8 mice compared to the SAMR1 mice. The total number of aggressive encounters, including attacks and offensive uprights, was found again significantly increased for the SAMP8 mice (Figures 3A-3C). Additionally, SAMP8 mice presented a tendency for the number of lateral threats to increase (Figure 3D). Accordingly, we found a significant increase in the time percentage of the overall aggression in SAMP8 mice (Figure 3E). Regarding the social interaction, the number of rearings was found reduced in the SAMP8 group in comparison with the SAMR1 group (Figure 3F). 

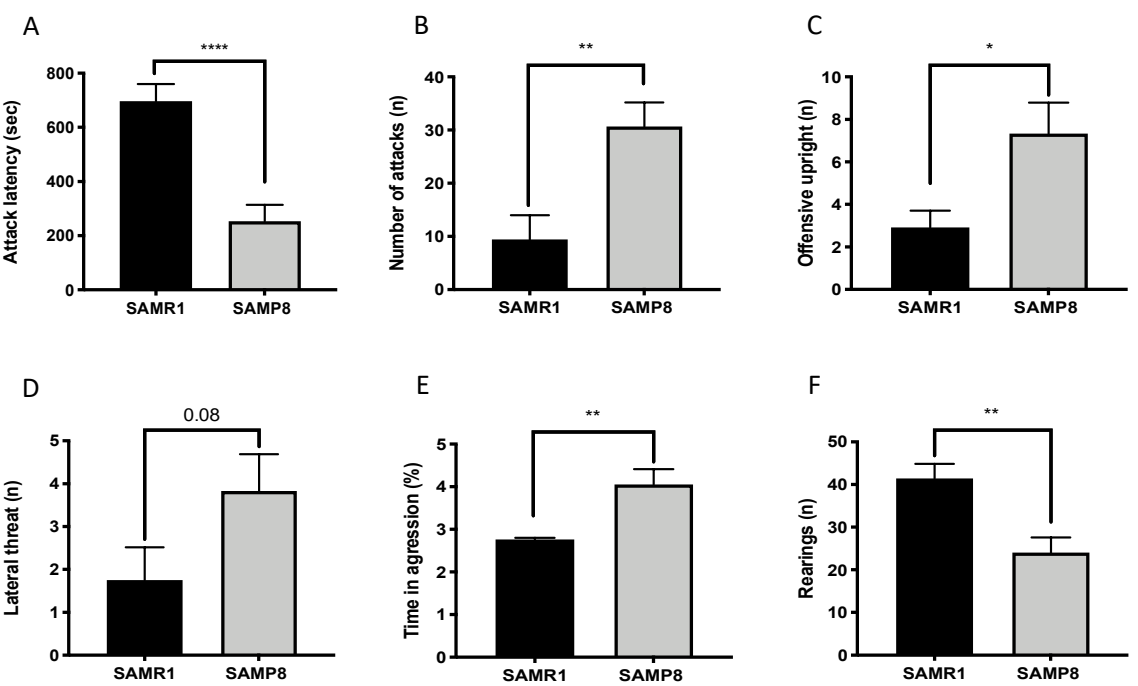

Figure 3. Results of Resident Intruder Test (RI) in males SAMR1 and SAMP8 mice at 12 months of age. Attack latency (A), number of attacks (B), number of offensive upright (C), number of lateral threat (D), time in aggression $(\mathrm{E})$, number of rearings $(\mathrm{F})$. Values represented are mean \pm Standard error of the mean (SEM); $\mathrm{n}=26$ (SAMR1 $\mathrm{n}=12$; SAMP8 $\mathrm{n}=14$ ). ${ }^{*} \mathrm{p}<0.05 ;{ }^{* *} \mathrm{p}<0.01 ;{ }^{* * * *} \mathrm{p}<0.0001$.

\subsection{Impaired cognitive capabilities and reduction in neurotrophins in aged SAMP8 male mice}

NORT revealed robust cognitive deficits in SAMP8 mice when compared to age and sex mated SAMR1. The weakened performance of SAMP8 mice in recognition of the new object resulted in a significant decrease in the DI, obtained after the $2 \mathrm{~h}$ short-memory test (Figure $4 \mathrm{~A}$ ). Furthermore, we evaluated in hippocampal tissue some important neurotrophins such as neurotrophin-3 (NT3) and brain-derived neurotrophic factor (BDNF). Strikingly, we found a significant reduction in NT3 gene expression as well as reduced BDNF protein levels in older SAMP8 group in comparison with SAMR1 (Figures 4B, 4C). 

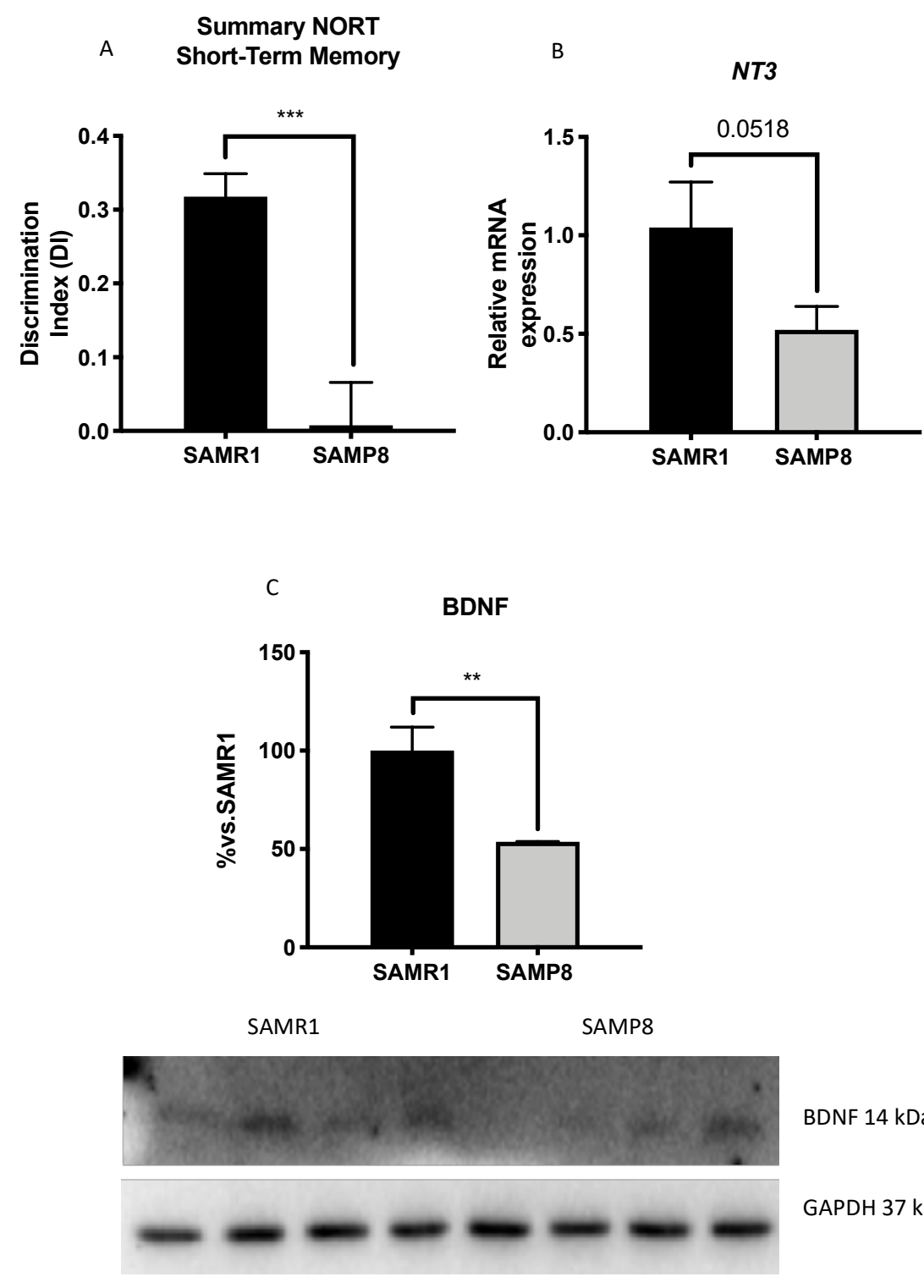

BDNF $14 \mathrm{kDa}$

GAPDH $37 \mathrm{kDa}$

Figure 4. Results of Novel Object Recognition Test (NORT) in males SAMR1 and SAMP8 at 12 months of age. Summary of short-term memory (A), representative gene expression for NT3 (B), and representative $\mathrm{WB}$ and quantification of $\operatorname{BDNF}(\mathrm{C})$. Values for NORT represented are mean \pm Standard error of the mean (SEM); $n=26$ (SAMR1 $n=12$; SAMP8 $n=14$ ). ${ }^{* *} p<0.001$. Values in bar graphs are adjusted to $100 \%$ for protein levels of the SAMR1 (SR1). Gene expression levels were determined by real-time PCR. Values are the mean \pm Standard error of the mean (SEM); $(n=4-6$ for each group). ${ }^{* *} \mathrm{p}<0.01$.

\subsection{Relationship between behaviour, cognition and molecular parameters}

There was a significant positive correlation between sociability expressed as time exploring the intruder mouse in TCT, and the DI evaluated in NORT [ $\mathrm{r} 2=0.4501, \mathrm{p}=0.0312]$, showing that decreased social interaction of SAMP8 is associated with cognitive decline (Figure 5A). Likewise, we found a significant positive correlation between the attack latency of the mice in the RI test and the DI [r2 = 0.5553, $\mathrm{p}=0.0049]$, suggesting that increased aggressive behaviour exhibited by aged SAMP8 mice is strongly related to the progression of cognitive impairment (Figure 5B). Regarding the anxiety state of the mice as assessed with OFT and EPM, a positive correlation between the distance travelled in center zone, and the DI was determined [ $\mathrm{r} 2=0.4117, \mathrm{p}=0.0569]$, whereas the time spent in open 
arms correlated negatively with the DI [ $\mathrm{r} 2=-0.3862, \mathrm{p}=0.0623]$. These latter correlations suggest that emotional disturbances of SAMP8 mice related to anxiety are associated with cognitive capabilities (Figures 5C, 5D). Furthermore, a positive correlation between BDNF protein levels and the DI was determined [ $\mathrm{r} 2=0.6834, \mathrm{p}=0.0617]$, as well as no correlation between NT3 gene expression and the DI was found [r2 $=0.3870, \mathrm{p}=0.2692]$ (Figures 5E, 5F).
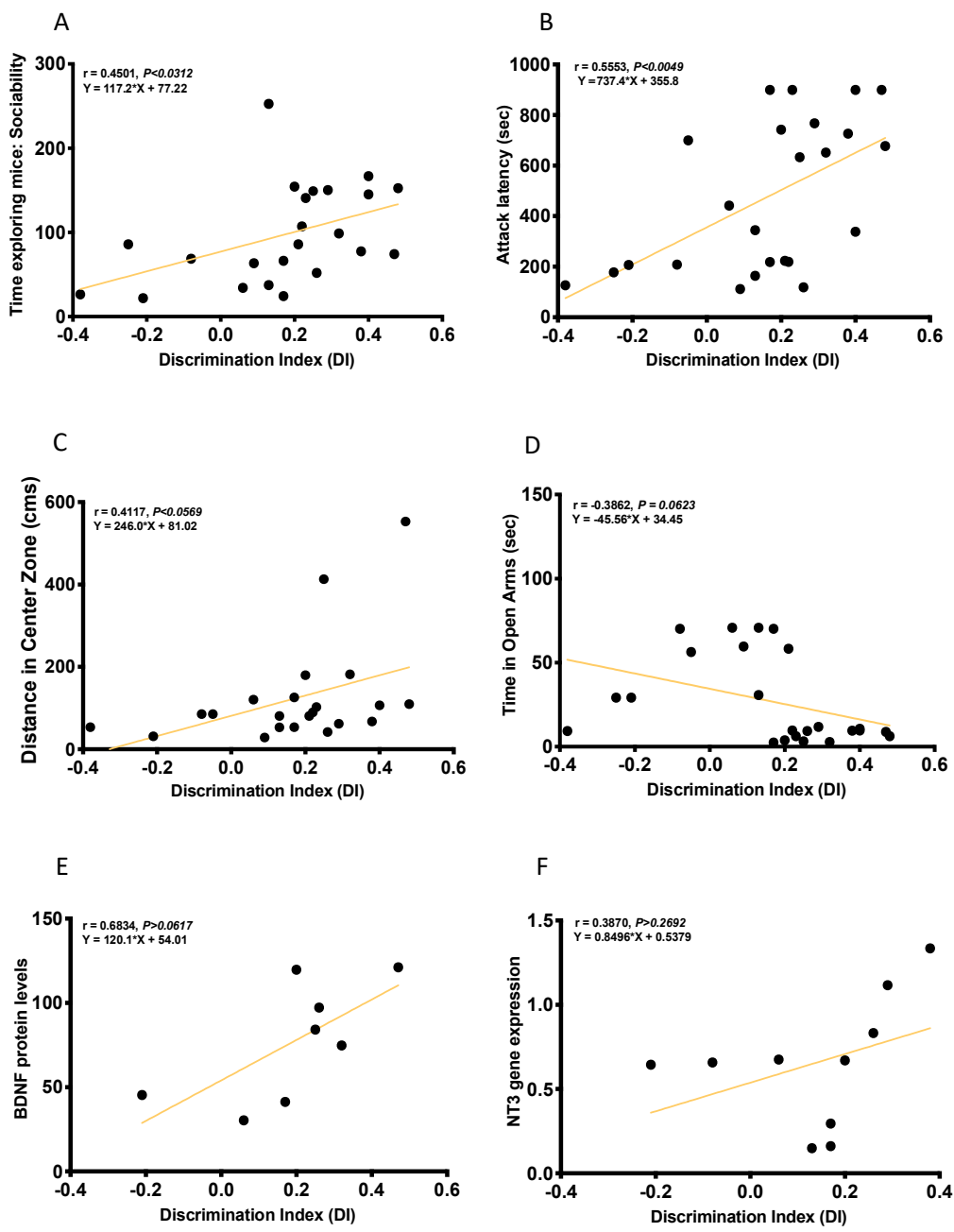

Figure 5. Correlations between time exploring mice: sociability (A), attack latency (B), distance in center zone (C), time in open arms (D), BDNF protein levels (E), and NT3 gene expression (F) with discrimination index (DI). Pearson correlations were performed between the behavioural test and molecular parameters in both groups $(n=4-12) . R^{2}$ and p-values were indicated on graphs. The levels of significance were $\mathrm{p}<0.05$.

\section{Discussion}

It has been reported that most $\mathrm{AD}$ patients present an association between cognitive dysfunction and BPSD. About $95 \%$ of AD patients have at least one NPS and syndromes, being the most frequent aggression (76.2\%) and depression (68\%) [24]. However, few studies are evaluating these symptoms that are unique to human beings [25], due to the lack of suitable animal models for being directly used for the study of BPSD and age-related emotional alterations. Additionally, to the best of our knowledge, there is not still an animal model that replicates identically all the psychiatric symptoms associated with AD. Indeed, transgenic animals are considered as an excellent tool to study alterations and symptoms related to familial AD but they are not good enough to evaluate sporadic aspects of the disease. The SAMP8 mouse is a well-characterized valid model for studying brain aging and AD [19]. Here, we investigated older male SAMP8 mice using a complete battery task to go 
deeper into the behavioural and emotional phenotype of the strain, focusing on the relationship between anxiety, aggression, sociability, cognitive deficits and two of the most important neurotrophins. Our results revealed significant correlations between emotional disturbances, cognitive performance and neurotrophins alterations in the SAMP8 mouse, resembling the clinical situation notably well.

Enhanced fear and anxiety-like behaviour is frequently associated with aging and AD in rodents $[26,27]$. Previous studies have reported ambiguous results regarding the anxiety-like behaviour of SAMP8 mice. Several lines of evidence show that SAMP8 mice show an age-associated emotional disorder characterized by reduced anxiety-like behaviour, while more recent studies have demonstrated that SAMP8 strain presents enhanced fear and anxiety linked to age-related cognitive decline in advanced age when compared to SAMR1 strain [23,28,29]. Accordingly, we obtained mixed results after the SAMP8 and SAMR1 groups were tested in OF and EPM. On the one hand, SAMP8 mice tended to spend less time in the center zone of the OFT and significantly more time in the border zone, results that corroborate the previously observed increased anxiety-like behaviour in the SAMP8 strain. On the other hand, SAMP8 spent significantly more time in the open arms of the EPM and less time in the center of the maze which could imply decreased anxiety levels in the SAMP8 mice in accordance with previous studies. However, data from the same behavioural test indicated that SAMP8 mice tended to spend more time in the closed arms of the maze as well when compared to the SAMR1 mice. These two latter observations could indicate that the parallel increases in the time spent in both open and closed maze's arms could be due to the increased locomotor activity that SAMP8 showed, letting unclear the interpretation of the EPM data as reduced anxiety levels for SAMP8 mice. Fear and anxiety-like behaviour has been linked to age-related cognitive deficits in SAMP8 mice $[15,17,30]$. Accordingly, in our study, we found significant correlations between the anxiety-related parameters and the cognitive performance confirmed the association of emotional alterations with memory impairment in aged SAMP8 males.

Social dysfunction observed in human AD patients has been observed in transgenic AD mice as well [27,31]. In our study, 12-month-old male SAMP8 mice exhibited social behaviour deficits by presenting no differences in the chambers preference and consequently interacting for less time with the intruder. Together these results are in line with previously published results which described decreased sociability in SAMP8 mice [23]. Jointly with social impairments, aggression is one of the most distressing consequences of dementia and of special significance given its direct influence on social functionality [32]. To our knowledge, the evaluation of the aggressive behaviour in old SAMP8 male mice has not been reported. Here, we demonstrated that 12-month-old SAMP8 males displayed increased aggressive behaviour by presenting decreased latency to attack and increased the total number of attacks. These results add to the diverse BPSD phenotype of aged SAMP8 mice, the presence of aggressive disturbances and corroborate the manifestation of aggression in advanced age and dementia in this mouse model. In addition, when the relationship between social or aggressive behaviour with cognitive performance was investigated, we found significant correlations. Social interaction positively correlated with cognitive performance, indicating that social behaviour impairment in SAMP8 may be implicated in cognitive impairment. Likewise, a significant negative correlation was determined between aggression and cognitive performance. As a consequence, our findings in SAMP8 mice support the consensus that aggression and social impairments in AD and age-related dementias are associated with the degree of cognitive impairment.

Ultimately, it has been described that BDNF and NT3 neurotrophins are involved in NPS such as anxiety, depression and aggressive behaviour [33]. In accordance with this, here, we demonstrated a consistent reduction in both BDNF protein levels and NT3 gene expression in older SAMP8 male mice. However, the correlation analysis only confirmed that BDNF levels correlate with cognitive decline and no correlation appeared between NT3 gene expression and cognitive decline. In line with these results, cumulative studies with AD animal models demonstrated that decreased BDNF levels lead to neuronal and synaptic dysfunction and eventual cognitive impairment [34]. Furthermore, regarding the NT3, the consensus establishes an unchanged mRNA and protein levels in the AD 
patients brain [35,36], confirming our lack of negative correlation between NT3 gene expression and cognitive decline.

To sum up, we demonstrated the presence of behavioural alterations in aged SAMP8 male mice including anxiety disorders, social impairment and aggressive behaviour, which correlated with the age-related cognitive deficits and neurotrophin alterations. These findings are in agreement with previous findings regarding the SAMP8 mouse model and give further support to this senescence model as a tool in gerontological research and shed new insight into the suitability of SAM strains for the study of non-cognitive alterations accompanied cognitive impairment and molecular changes in senescence.

\section{Materials and Methods}

\subsection{Subjects}

A total of 26 males SAMP8 and SAMR1 mice aged 12-month-old were used to perform behavioural experiments. The animals were divided into two groups: SAMP8 $(n=14)$ and SAMR1 $(\mathrm{n}=12)$. Animals had free access to food and water and were maintained under standard temperature conditions $\left(22 \pm 2^{\circ} \mathrm{C}\right)$ and $12 \mathrm{~h}$ light-dark cycle (300 lux/0 lux) in plastic cages with wood shaving bedding.

All experimental procedures involving animals were followed the standard ethical guidelines of European Communities Council Directive 86/609/EEC and by the Institutional Animal Care and by Generalitat de Catalunya (10291, approved 1/28/2018). All efforts were made to minimize the number of mice used and their suffering.

\subsection{Behavioural experiments}

\subsubsection{Open Field Test}

Emotional alterations were evaluated by the Open Field (OF) test and performed using a white plywood apparatus $(50 \times 50 \times 25 \mathrm{~cm})$, as previously described [37]. Apparatus' ground was divided into center and peripheral areas. Behaviour was evaluated with SMART ver. 3.0 software (Panlab, Cornellà, Barcelona, Spain), and each trial was recorded for later analysis, with the use of a camera fixed to the ceiling, above the apparatus. Mice were placed at the centre of the open field and allowed to explore the apparatus for $5 \mathrm{~min}$. After the session, mice were returned to their home cages, and the open field was cleaned with $70 \%$ ethanol and allowed to dry between sessions. The parameters scored included Locomotor Activity calculated as the sum of total distance travelled in $5 \mathrm{~min}$ as well as the time spent in Centre and Periphery zone, and the number of rearings, defecations, and urinations.

\subsubsection{Elevated Plus Maze}

The anxiety-like behaviour was assessed by The Elevated Plus Maze (EPM) apparatus was performed in an apparatus which consisted of two open arms $(30 \times 5 \times 15 \mathrm{~cm})$ and two closed arms $(30 \times 5 \times 15 \mathrm{~cm})$ as described previously [37]. The arms radiated from a central platform $(5 \times 5 \mathrm{~cm})$. Behaviour was scored with SMART ver. 3.0 software (Panlab, Cornellà, Barcelona, Spain), and each trial was recorded for later analysis, utilizing a camera fixed to the ceiling above the apparatus. To initiate the test session, the mice were placed on the central platform, facing an open arm, and allowed to explore the apparatus for $5 \mathrm{~min}$. After the session, the mice were returned to their home cages, and the EPM apparatus was cleaned with $70 \%$ ethanol and allowed to dry between tests. Parameters recorded included total distance travelled during the 5-min test, time spent in open arms, closed arms and centre as well as the number of rearings.

\subsubsection{Novel Object Recognition Test (NORT)}

Object novel recognition was performed in a 90-degree, two-arm, 25-cm-long, 20-cm- high maze as previously described [38]. The walls could be easily removed for cleaning. Briefly, mice were 
individually habituated to the apparatus for $10 \mathrm{~min}$ for 3 days. On day 4, they were submitted to a 10-min acquisition trial (familiarization phase), during which, they were placed in the maze and allowed to explore two identical novel objects $(\mathrm{A}+\mathrm{A}$ or $\mathrm{B}+\mathrm{B})$ placed at the end of each arm. 10-min retention trials (test phase) occurred $2 \mathrm{~h}$ and $24 \mathrm{~h}$ after the familiarization phase. During test phase, one of the two identical objects were replaced by a novel one and the time spent exploring the new object (TN) and the old one (TO) were evaluated manually. The discrimination index (DI) was calculated as (TN-TO/TN+TO). To avoid object preference biases, objects $\mathrm{A}$ and $\mathrm{B}$ were counterbalanced so that one half of the animals in each experimental group were first exposed to object $\mathrm{A}$ and then to object $\mathrm{B}$, whereas the other one half first saw object $\mathrm{B}$ and then object $\mathrm{A}$ was presented. The maze, the surface, and the objects were cleaned with $70 \%$ ethanol between the animals' trials to eliminate olfactory cues.

\subsubsection{Three Chamber Test (TCT)}

The Three-Chamber test assesses cognition through sociability and interest in social novelty [39]. The TCT was performed using a box $(15 \times 15 \times 20 \mathrm{~cm})$ divided in three equally dimensioned rooms with openings among them. Testing had a duration of 20 minutes and occurred in two phases within the TCT apparatus. The animal was placed in the center of the box and allowed to explore the three chambers for $5 \mathrm{~min}$ (Habituation phase). The time spent in each room during the habituation phase was measured manually. Afterwards, an intruder (same sex and age) was added to one of the rooms in a metal cage and behaviour was recorded for $10 \mathrm{~min}$. In this phase, both the time spent in each room and the time interacting with the intruder (e.g., sniffing, grooming) were measured. The TCT apparatus, the surface, and the metal cages were cleaned with $70 \%$ ethanol between the animals' trials to eliminate olfactory cues.

\subsubsection{Resident-Intruder Test}

The Resident-Intruder (RI) test was performed in order to evaluate the aggressive behaviour exhibited by the animals as described previously [40]. Briefly, the test subjects (residents) were isolated in separate cases for 7 days prior to the performance of the test. On the test day, an intruder animal was introduced in the resident home cage. The entire 20-min session was video-recorded for later analysis. Aggressive behaviour of the residents was evaluated as latency for the first attack, offensive uprights, lateral threats, total number of attacks as well as the overall time (\%) of aggression, which was measured manually. Social interaction during the 20-min session was evaluated as a number of rearings.

\subsection{Molecular studies}

\subsubsection{Western blotting}

For Western Blotting (WB), aliquots of $15 \mu \mathrm{g}$ of hippocampal protein were used. Protein samples were separated by SDS-PAGE $(8-12 \%)$ and transferred into Polyvinylidene difluoride (PVDF) membranes (Millipore, Billerica, MA, USA). Afterwards, membranes were blocked in $5 \%$ non-fat milk in $0,1 \%$ Tween 20 TBS (TBS-T) for $1 \mathrm{~h}$ at room temperature, followed by overnight incubation at $4^{\circ} \mathrm{C}$ with the primary antibodies listed in Table S1.

Afterwards, membranes were washed and incubated with secondary antibodies for $1 \mathrm{~h}$ at room temperature. Immunoreactive proteins were viewed with a chemiluminescence-based detection kit, following the manufacturer's protocol (ECL Kit; Millipore, Billerica, MA, USA) and digital images were acquired using a ChemiDoc XRS+ System (BioRad, Hercules, CA, USA). Semi-quantitative analyses were carried out using ImageLab software (BioRad, Hercules, CA, USA) and results were expressed in Arbitrary Units (AU), considering control protein levels as 100\%. Protein loading was routinely monitored by immunodetection of GAPDH. 


\subsubsection{RNA extraction and gene expression determination}

Total RNA isolation was carried out using TRIsure ${ }^{\mathrm{TM}}$ reagent following the manufacturer's instructions (Bioline Reagent, London, UK). RNA content in the samples was measured at $260 \mathrm{~nm}$, and the purity of the samples was determined by the A260/280 and A260/230 ratio in a NanoDrop ${ }^{\mathrm{TM}}$ ND-1000 (Thermo Scientific). Reverse transcription-Polymerase chain reaction (RT-PCR) was performed as follows: $2 \mu \mathrm{g}$ of messenger RNA (mRNA) was reverse-transcribed using the High Capacity cDNA Reverse Transcription kit (Applied Biosystems, Foster City, CA, USA). Real-time quantitative PCR (qPCR) was employed to quantify the mRNA expression of NT3. The primers were those listed in Table S2.

For SYBER Green, real-time PCR was performed in the Step One Plus Detection System (Applied Biosystems) employing the SYBR Green PCR Master Mix (Applied Biosystems). Each reaction mixture contained $6.75 \mu \mathrm{L}$ of cDNA, whose concentration was $2 \mu \mathrm{g}, 0.75 \mu \mathrm{L}$ of each primer (whose concentration was $100 \mathrm{nM})$, and $6.75 \mu \mathrm{L}$ of SYBR Green PCR Master Mix (2X).

Data were analyzed by using the comparative Cycle threshold $(\mathrm{Ct})$ method $(\Delta \Delta \mathrm{Ct})$, where the $\odot$ actin transcript level was used to normalize differences in sample loading and preparation. Each sample $(n=4-6)$ was analyzed in duplicate, and results represented the $n$-fold difference of transcript levels among different samples.

\subsection{Statistical Analysis}

Data are expressed as the mean \pm Standard error of the mean (SEM). Statistical analyses were performed using unpaired Student's t test with GraphPad Prism ver. 8 for Mac. Correlations among different parameters were analyzed with Pearson's correlation. Statistical significance was considered when $\mathrm{p}$-values were $<0.05$. Statistical outliers were discriminated using Grubbs' test and were removed from the analysis.

Supplementary Materials: Table S1. Antibodies used in Western blot studies. Table S2. Primers used in qPCR. Table S3. Parameters measured in the Elevated Plus Maze (EPM) in male SAMR1 and SAMP8 mice at 12 months of age. (n): number of events. Results are expressed as a mean \pm Standard error of the mean $(\mathrm{SEM}) .{ }^{*} \mathrm{p}<0.05$; ${ }^{* *} \mathrm{p}<0.01 ;{ }^{* * *} \mathrm{p}<0.001 ;{ }^{* * * *} \mathrm{p}<0.0001$. Table S4. Parameters measured in the Open Field Test (OFT) in male SR1 and SP8 mice at 12 months of age. (n): number of events. Results are expressed as a mean \pm Standard error of the mean (SEM). ${ }^{*} \mathrm{p}<0.05 ;{ }^{* *} \mathrm{p}<0.01 ;{ }^{* * * *} \mathrm{p}<0.0001$.

Author Contributions: F.V. and J. C.-A. contributed equally. C. G.-F., A.-M. C., and M. P. designed the study. C. G.-F, F. V. and J. C.-A. carried out the behaviour and cognition studies and molecular parameters determination. F. V., J. C.-A., V.P.-A., A.-M.C., D. O.-S., C.G.-F., and M.P. contributed to writing the manuscript. All authors have read and approved the final version of the manuscript.

Funding: This study was funded by Ministerio de Economía y Competitividad of Spain (PID2019-106285RB). F.V., J.C.A., C.G.-F. and M.P. belong to 2017SGR106 (AGAUR, Catalonia). Financial support for F.V. (University of Barcelona, APIF_2017), J.C.-A. (FPI fellowship/contract_MDM-2017-0729-19-2) from Plan Nacional de Investigación, Spain, co-financed by FEDER and V.P.-Á. (University of Guadalajara, V/2014/2016).

Conflicts of Interest: The authors of this manuscript have no conflict of interests to declare.

\section{Abbreviations}

$\begin{array}{ll}\text { AD } & \text { Alzheimer's disease } \\ \text { ANOVA } & \text { One-way analysis of variance } \\ \text { BDNF } & \text { Bran-derived neurotrophic factor } \\ \text { BPSD } & \text { Behavioural and psychological symptoms of dementia } \\ \text { cDNA } & \text { Complementary DNA } \\ \text { CNS } & \text { Central nervous system } \\ \text { Ct } & \text { Cycle threshold } \\ \text { DI } & \text { Discrimination index } \\ \text { EPM } & \text { Elevated pluz maze } \\ \text { GAPDH } & \text { Glyreladehyde-3-phosphate dehydrogenase }\end{array}$




$\begin{array}{ll}\text { mRNA } & \text { messenger RNA } \\ \text { ND } & \text { Neuropsychiatric disorders } \\ \text { NORT } & \text { Novel object recognition test } \\ \text { NPS } & \text { Neuropsychiatric symptoms } \\ \text { NT3 } & \text { Neurotrophin-3 } \\ \text { OD } & \text { Optical density } \\ \text { PCR } & \text { Polymerase chain reaction } \\ \text { PVDF } & \text { Polyvinylidene difluoride } \\ \text { RNA } & \text { Ribonucleic acid } \\ \text { qPCR } & \text { Real-time quantitative PCR } \\ \text { RI } & \text { Resident-intruder } \\ \text { SAMP8 } & \text { Senescence-accelerated mouse prone 8 } \\ \text { SAMR1 } & \text { Senescence-accelerated mouse resistant 1 } \\ \text { SEM } & \text { Standard error of the mean } \\ \text { SDS-PAGE } & \text { Sodium dodecyl sulfate-polyacrylamide } \\ \text { TBS } & \text { Tris-buffered saline } \\ \text { TBS-T } & \text { Tris-buffered saline tween 20 } \\ \text { TCT } & \text { Three chamber test } \\ \text { TN } & \text { Time with new object } \\ \text { TO } & \text { Time with old object } \\ \text { WB } & \text { Western blot } \\ \end{array}$

\section{References}

1. López-Otín, C.; Blasco, M.A.; Partridge, L.; Serrano, M.; Kroemer, G. The hallmarks of aging. Cell 2013, 153, 1194-217.

2. Franceschi, C.; Garagnani, P.; Morsiani, C.; Conte, M.; Santoro, A.; Grignolio, A.; Monti, D.; Capri, M.; Salvioli, S. The continuum of aging and age-related diseases: Common mechanisms but different rates. Front. Med. 2018, 5, 1.

3. Zhao, Q.F.; Tan, L.; Wang, H.F.; Jiang, T.; Tan, M.S.; Tan, L.; Xu, W.; Li, J.Q.; Wang, J.; Lai, T.J.; et al. The prevalence of neuropsychiatric symptoms in Alzheimer's disease: Systematic review and meta-analysis. J. Affect. Disord. 2016, 190, 264-271.

4. Stephan, Y.; Sutin, A.R.; Luchetti, M.; Terracciano, A. Subjective age and risk of incident dementia: Evidence from the National Health and Aging Trends survey. J. Psychiatr. Res. 2018, 100, 1-4.

5. Blennow, K.; de Leon, M.J.; Zetterberg, H. Alzheimer's disease. Lancet (London, England) 2006, 368, 387403.

6. Finkel, S.I. Behavioral and psychological symptoms of dementia (BPSD): a current focus for clinicians, researchers, caregivers, and governmental agencies. In Contemporary Neuropsychiatry; Springer, 2001; pp. 200-210.

7. Shinosaki, K.; Nishikawa, T.; Takeda, M. Neurobiological basis of behavioral and psychological symptoms in dementia of the Alzheimer type. Psychiatry Clin. Neurosci. 2000, 54, 611-620.

8. Hersch, E.C.; Falzgraf, S. Management of the behavioral and psychological symptoms of dementia. Clin. Interv. Aging 2007, 2, 611.

9. Bidzan, L.; Bidzan, M.; Pąchalska, M. Aggressive and impulsive behavior in Alzheimer's disease and progression of dementia. Med. Sci. Monit. Int. Med. J. Exp. Clin. Res. 2012, 18, CR182.

10. Lawlor, B. Managing behavioural and psychological symptoms in dementia. Br. J. Psychiatry 2002, 181, 463-465.

11. Toot, S.; Swinson, T.; Devine, M.; Challis, D.; Orrell, M. Causes of nursing home placement for older people with dementia: a systematic review and meta-analysis. Int. Psychogeriatrics 2017, 29, 195-208.

12. J Allen, S.; J Watson, J.; Dawbarn, D. The neurotrophins and their role in Alzheimer's disease. Curr. Neuropharmacol. 2011, 9, 559-573.

13. Narisawa-Saito, M.; Wakabayashi, K.; Tsuji, S.; Takahashi, H.; Nawa, H. Regional specificity of alterations in NGF, BDNF and NT-3 levels in Alzheimer's disease. Neuroreport 1996, 7, 2925-2928.

14. Takeda, T.; Hosokawa, M.; Takeshita, S.; Irino, M.; Higuchi, K.; Matsushita, T.; Tomita, Y.; Yasuhira, K.; Hamamoto, H.; Shimizu, K. A new murine model of accelerated senescence. Mech. Ageing Dev. 1981, 17, 183-194. 
15. Takeda, T. Senescence-accelerated mouse (SAM) with special references to neurodegeneration models, SAMP8 and SAMP10 mice. Neurochem. Res. 2009, 34, 639-59.

16. Morley, J.E.; Armbrecht, H.J.; Farr, S.A.; Kumar, V.B. The senescence accelerated mouse (SAMP8) as a model for oxidative stress and Alzheimer's disease. Biochim. Biophys. Acta - Mol. Basis Dis. 2012, 1822, 650-656.

17. Pallàs, M. Senescence-Accelerated Mice P8: A Tool to Study Brain Aging and Alzheimer's Disease in a Mouse Model. ISRN Cell Biol. 2012, 2012, 1-12.

18. Pallàs, M. Senescence-Accelerated Mice P8: A Tool to Study Brain Aging and Alzheimer's Disease in a Mouse Model. ISRN Cell Biol. 2012, 2012, 1-12.

19. Morley, J.E.; Farr, S.A.; Kumar, V.B.; Armbrecht, H.J. The SAMP8 Mouse : A Model to Develop Therapeutic Interventions for Alzheimer's Disease. 2012, 1123-1130.

20. Miyamoto, M. Characteristics of age-related behavioral changes in senescence-accelerated mouse SAMP8 and SAMP10. Exp. Gerontol. 1997, 32, 139-148.

21. Takeda, T.; Hosokawa, M.; Higuchi, K.; Hosono, M.; Akiguchi, I.; Katoh, H. A novel murine model of aging, Senescence-Accelerated Mouse (SAM). Arch. Gerontol. Geriatr. 1994, 19, 185-192.

22. Miyamoto, M. Emotional disorders and memory deficits in senescence-accelerated mice, SAMP8 and SAMP10. In Proceedings of the International Congress Series; Elsevier, 2004; Vol. 1260, pp. 99-106.

23. Meeker, H.C.; Chadman, K.K.; Heaney, A.T.; Carp, R.I. Assessment of social interaction and anxiety-like behavior in senescence-accelerated-prone and-resistant mice. Physiol. Behav. 2013, 118, 97-102.

24. Youn, J.C.; Lee, D.Y.; Jhoo, J.H.; Kim, K.W.; Choo, I.H.; Woo, J.I. Prevalence of neuropsychiatric syndromes in Alzheimer's disease (AD). Arch. Gerontol. Geriatr. 2011, 52, 258-263.

25. Cerejeira, J.; Lagarto, L.; Mukaetova-Ladinska, E. Behavioral and psychological symptoms of dementia. Front. Neurol. 2012, 3, 73.

26. Godbout, J.P.; Chen, J.; Abraham, J.; Richwine, A.F.; Berg, B.M.; Kelley, K.W.; Johnson, R.W. Exaggerated neuroinflammation and sickness behavior in aged mice after activation of the peripheral innate immune system. FASEB J. 2005, 19, 1329-1331.

27. Filali, M.; Lalonde, R.; Rivest, S. Cognitive and non-cognitive behaviors in an APPswe/PS1 bigenic model of Alzheimer's disease. Genes, Brain Behav. 2009, 8, 143-148.

28. Miyamoto, M.; Takahashi, H.; Ohta, H.; Sakamoto, J. Animal Model of Brain Aging: SenescenceAccelerated Mouse (SAM). CNS Drug Rev. 1998, 4, 361-375.

29. Griñán-Ferré, C.; Corpas, R.; Puigoriol-Illamola, D.; Palomera-Ávalos, V.; Sanfeliu, C.; Pallàs, M. Understanding Epigenetics in the Neurodegeneration of Alzheimer's Disease: SAMP8 Mouse Model. J. Alzheimer's Dis. 2018, 62.

30. Flood, J.F.; Farr, S.A.; Uezu, K.; Morley, J.E. Age-related changes in septal serotonergic, GABAergic and glutamatergic facilitation of retention in SAMP8 mice. Mech. Ageing Dev. 1998, 105, 173-188.

31. Kosel, F.; Munoz, P.T.; Yang, J.R.; Wong, A.A.; Franklin, T.B. Age-related changes in social behaviours in the 5xFAD mouse model of Alzheimer's disease. Behav. Brain Res. 2019, 362, 160-172.

32. Jackson, J.L.; Mallory, R. Aggression and violence among elderly patients, a growing health problem 2009.

33. L Neto, F.; Borges, G.; Torres-Sanchez, S.; A Mico, J.; Berrocoso, E. Neurotrophins role in depression neurobiology: a review of basic and clinical evidence. Curr. Neuropharmacol. 2011, 9, 530-552.

34. Peng, S.; Garzon, D.J.; Marchese, M.; Klein, W.; Ginsberg, S.D.; Francis, B.M.; Mount, H.T.J.; Mufson, E.J.; Salehi, A.; Fahnestock, M. Decreased brain-derived neurotrophic factor depends on amyloid aggregation state in transgenic mouse models of Alzheimer's disease. J. Neurosci. 2009, 29, 9321-9329.

35. Durany, N.; Michel, T.; Kurt, J.; Cruz-Sánchez, F.F.; Cervós-Navarro, J.; Riederer, P. Brain-derived neurotrophic factor and neurotrophin-3 levels in Alzheimer's disease brains. Int. J. Dev. Neurosci. 2000, 18, 807-813.

36. Hock, C.; Heese, K.; Müller-Spahn, F.; Hulette, C.; Rosenberg, C.; Otten, U. Decreased trkA neurotrophin receptor expression in the parietal cortex of patients with Alzheimer's disease. Neurosci. Lett. 1998, 241, 151-154.

37. Griñan-Ferré, C.; Palomera-Ávalos, V.; Puigoriol-Illamola, D.; Camins, A.; Porquet, D.; Plá, V.; Aguado, F.; Pallàs, M. Behaviour and cognitive changes correlated with hippocampal neuroinflammaging and neuronal markers in female SAMP8, a model of accelerated senescence. Exp. Gerontol. 2016, 80.

38. Griñán-Ferré, C.; Codony, S.; Pujol, E.; Yang, J.; Leiva, R.; Escolano, C.; Puigoriol-Illamola, D.; CompanysAlemany, J.; Corpas, R.; Sanfeliu, C. Pharmacological inhibition of soluble epoxide hydrolase as a new therapy for Alzheimer's Disease. bioRxiv 2019, 605055.

39. Companys-Alemany, J.; Turcu, A.L.; Bellver-Sanchis, A.; Loza, M.I.; Brea, J.M.; Canudas, A.M.; Leiva, R.; Vázquez, S.; Pallàs, M.; Griñán-Ferré, C. A Novel NMDA Receptor Antagonist Protects against Cognitive Decline Presented by Senescent Mice. Pharmaceutics 2020, 12, 284. 
40. Maes, T.; Mascaró, C.; Rotllant, D.; Lufino, M.M.P.; Estiarte, A.; Guibourt, N.; Cavalcanti, F.; Griñan-Ferré, C.; Pallàs, M.; Nadal, R. Modulation of KDM1A with vafidemstat rescues memory deficit and behavioral alterations. PLoS One 2020, 15, e0233468. 